\title{
KIR and HLA-C: Immunogenetic regulation of human birth weight
}

\author{
Lydia E. Farrel1 ${ }^{1,2}$, Susan E. Hiby ${ }^{1,2}$, Richard Apps ${ }^{3,4}$, Olympe Chazara ${ }^{1,2}$, Lill Trogstad ${ }^{5}$, \\ Håkon K. Gjessing ${ }^{6}$, Per Magnus ${ }^{6}$, Mary Carrington ${ }^{3,4}$ and Ashley Moffett ${ }^{1,2}$ \\ 1) Department of Pathology, University of Cambridge, Cambridge CB2 1QP, United Kingdom \\ 2) Centre for Trophoblast Research, University of Cambridge, Cambridge CB2 1QP, United Kingdom \\ 3) Cancer and Inflammation Program, Laboratory of Experimental Immunology, Leidos Biomedical Research \\ Inc., Frederick National Laboratory, Frederick, MD 21702, USA \\ 4) Ragon Institute of Massachusetts General Hospital, Massachusetts Institute of Technology and Harvard \\ University, Cambridge, MA 02139, USA \\ 5) Division of Infectious Disease Control, Norwegian Institute of Public Health, 0403 Oslo, Norway \\ 6) Division of Epidemiology, Norwegian Institute of Public Health, 0403 Oslo, Norway \\ Correspondence: Lydia E. Farrell, e-mail lf284@cam.ac.uk
}

\begin{abstract}
Pregnancies resulting in very small or very large babies are at higher risk of obstetric complications with increased morbidity for both mother and baby. Using data from the Medical Birth Registry of Norway we have shown how human birth weight is still subject to stabilizing selection. Particular combinations of maternal/fetal immune genes have been implicated in pregnancies resulting in a low birth weight baby $\left(<5^{\text {th }}\right.$ birth weight centile). More specifically, an inhibitory maternal KIRAA genotype with a paternally derived fetal $H L A-C 2$ ligand. At the other end of the birth weight spectrum the presence of an activating maternal KIR2DS1 gene is associated with increased birth weight in linear or logistic regression analyses of all pregnancies $>5^{\text {th }}$ centile $(\mathrm{p}=0.005, \mathrm{OR}=2.65$ ). Thus, inhibitory maternal KIR combined with fetal $H L A$ $C 2$ is more frequently associated with low birth weight, whereas activating maternal $K I R$ with fetal $H L A$ $C 2$ ligand is associated with increasing birth weight. Our findings using the MoBa cohort have replicated the association of KIR and $H L A-C$ seen in poor placentation, and confirm the importance of maternal/fetal immune gene interactions in determining the outcome of pregnancy.
\end{abstract}

This is an open access article distributed under the Creative Commons Attribution Licence, which permits unrestricted use, distribution, and reproduction in any medium, provided the original work is properly cited.

Our work aims to understand how the immune system contributes to successful pregnancy. The placenta forms the interface between the mother and baby in the uterus. Fetal extravillous trophoblast cells (EVT) invade into the maternal decidua where they remodel uterine spiral artery walls converting them into high conductance vessels, thus securing a good blood supply to the feto-placental unit [1]. Insufficient transformation of the maternal spiral arteries results in poor placental development which consequently gives rise to disorders of pregnancy such as pre-eclampsia, fetal growth restriction (FGR), still birth and recurrent miscarriage [2-4]. Excessive invasion by EVT can also be detrimental in cases such as placenta accreta. High birth weight is potentially dangerous to both mother and baby through prolonged obstructed labour $[5,6]$ with risk of asphyxia and injury in the baby [7-9], and lacerations and post-partum haemorrhage in the mother $[5,9]$. It is therefore crucially important that this process is carefully regulated maintaining a balance between over and under invasion. Using data from 795,068 first pregnancies resulting in a live birth from the Medical Birth Registry of Norway, we plotted the distribution of birth weight against frequency of transfer to special care baby unit [10]. This illustrated clearly the risk of morbidity in neonates at either end of the birth weight spectrum, even in a modern medicalised setting (Fig. 1).
Large numbers of a specialised type of lymphocyte known as uterine NK cells are found in the decidua during placentation [11]. Uterine NK cells express Killer cell Ig-like Receptors (KIR) and fetal EVT express their cognate ligand HLA-C, the only classical HLA class I molecule found on trophoblast [12]. We have proposed that this maternal KIR/fetal HLA-C interaction functions to mediate uterine $\mathrm{NK}$ cell control of trophoblast invasion [11,13-15].

This receptor ligand interaction is unusual in that both KIR and HLA are highly polymorphic gene systems. They also segregate independently and are encoded on separate chromosomes. KIR genotypes vary with content, copy number and at allelic variation at individual KIR loci. Around 500 different genotypes have already been described to date [16]. To simplify this complexity, KIR haplotypes are classified as either $A$ or $B$ based on gene content. The KIR $A$ haplotype is highly stable, varying little at the gene content level and carrying fewer genes. Notably it encodes KIR2DL1 and KIR2DL3, both inhibitory receptors for HLA-C. The KIR $B$ haplotype is much more variable with the potential to encode inhibitory KIR2DL1 and $K I R 2 D L 2$, and also activating KIR2DS1, all of which bind HLA-C [11].

$H L A-C$ alleles can be subdivided into two groups $\mathrm{C} 1$ and $\mathrm{C} 2$ based on a dimorphism at position 80 of the 


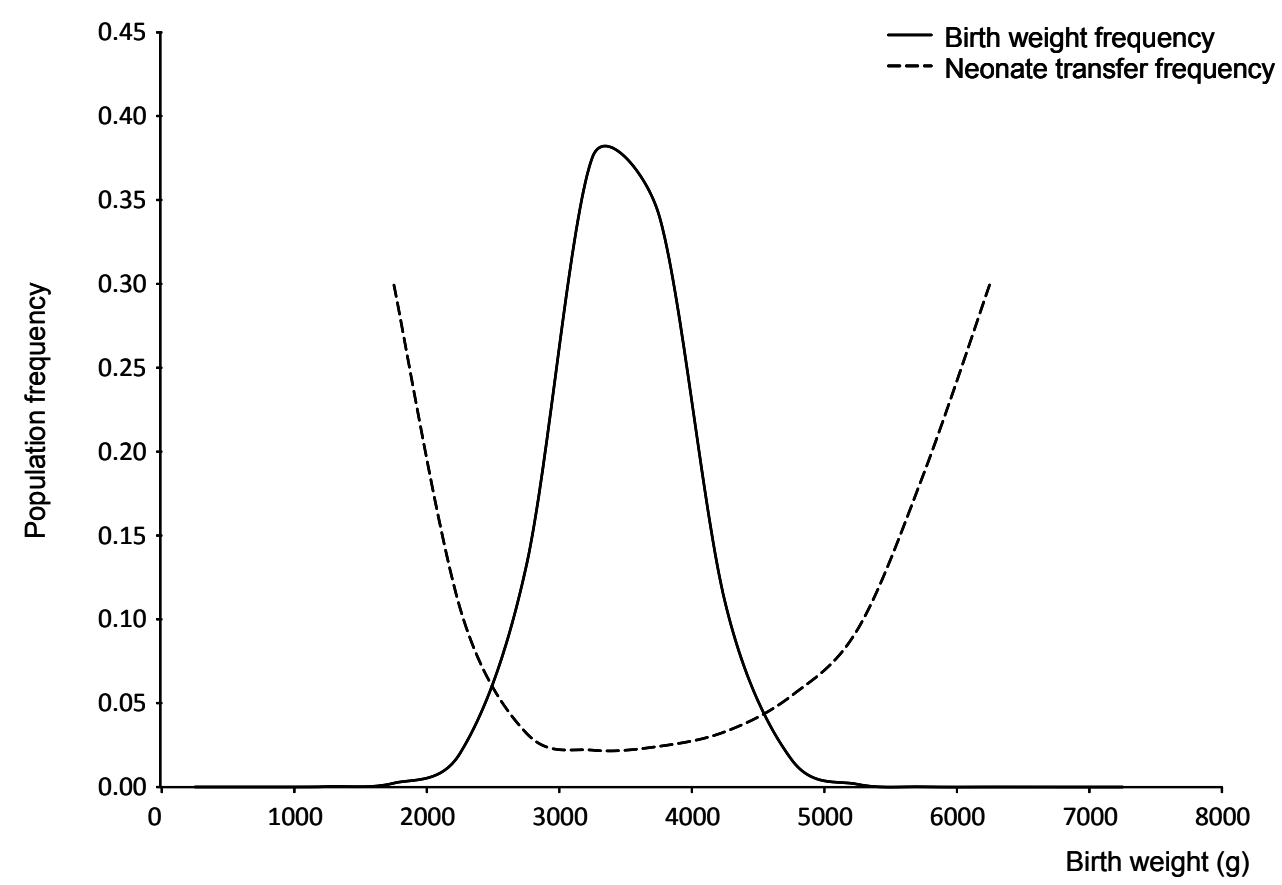

Figure 1. Distribution of birth weights in the Norwegian population with percentage of babies transferred to the special care baby unit for the years 1967-2010 $(\mathrm{n}=795,068)$. (Originally published in Journal of Immunology: Hiby $\mathrm{S}$, Apps R, Chazara O, et al. Maternal KIR in combination with paternal HLA-C2 regulate human birth weight. $J$. Immunol 2014; 192: 5069-5073. Copyright (C) [2014] The American Association of Immunologists, Inc.)

$\alpha 1$ domain [17]. In this way KIR distinguish between $\mathrm{C} 1$ and $\mathrm{C} 2$ as mutually exclusive epitopes (Table 1 ).

To determine if maternal KIR and fetal HLA-C variation is important in pregnancy we have looked for consistent genetic associations of particular maternal $K I R$ and fetal HLA-C combinations in a range of obstetric disorders (pre-eclampsia, FGR, and recurrent miscarriage) all of which result from defective placentation. All these conditions share a genetic association characterised by mothers with two KIR $A$ haplotypes ( $A A$ genotype), combined with an $H L A-C 2$ group allele in the fetus, particularly when the fetal $C 2$ is paternally derived. Conversely, women who carry a KIR $B$ haplotype encoding KIR2DS1, the activating receptor for $\mathrm{C} 2$, are significantly protected from these disorders when a fetal $C 2$ is present $[11,18,19]$.

Because we were intrigued by the high fetal and maternal mortality and morbidity seen in pregnancies with high birth weights [5-9] we went on to question what maternal KIR and fetal $H L A-C$ combinations were associated with these pregnancies.

Mother and baby DNA pairs were genotyped for $K I R$ and $H L A-C$ from two separate cohorts. From our United Kingdom cohort we analysed 747 preeclamptic pregnancies, 118 pregnancies with FGR $\left(\leq 5^{\text {th }}\right.$ birth weight centile) and 404 normal pregnancies [10]. Selected from the MoBa cohort [20] were 995 normal (including $66>90^{\text {th }}$ birth weight centile), and 141 pre-eclamptic first pregnancies.

Replicating our previous findings from the UK, increased maternal KIR AA and low KIR2DSI frequencies associated with pregnancies with low birth weight and/or pre-eclampsia in the MoBa cohort. Conversely, high birth weight pregnancies $\left(\geq 90^{\text {th }}\right.$ centile) had low KIR AA and high KIR2DSI frequencies [10].

The effect of KIR2DS1 on birth weight was tested in both categorical and continuous analysis. Using birth weight in grams as a continuous variable, the presence of a maternal KIR2DS1 conferred an average birth weight increase of $78 \mathrm{~g}(\mathrm{p}=0.005)$ in a linear regression model. The frequency of maternal KIR2DSI was significantly higher in pregnancies with high compared with median birth weight in categorical analysis (Table 2, [10]).

The effect of KIR $A A$ genotypes on pre-eclampsia and FGR was previously observed particularly in pregnancies with a fetus carrying a paternally derived $C 2$. This was shown by both categorical and continuous analysis across the birth weight spectrum. The effect of maternal KIR2DSI on birth weight is thus dependent on the presence of fetal $C 2$, particularly paternally derived fetal $C 2$.

In other words the presence of fetal $C 2$ amplifies the effect of maternal KIR2DSI. When the fetus has more $C 2$ groups than the mother $(\mathrm{Cl} / \mathrm{C} 2$ fetus with $\mathrm{Cl} / \mathrm{Cl}$ mother, and $\mathrm{C} 2 / \mathrm{C} 2$ fetus with $\mathrm{Cl} / \mathrm{C} 2$ mother), an average increase of $245 \mathrm{~g}(\mathrm{p}=0.002)$ was seen. Furthermore, presence or absence of KIR2DS1 as a categorical variable when combined with more $C 2$ in the fetus showed that maternal KIR2DSI has a significant effect when the fetus is carrying more $C 2$ than the mother, (OR 2.93, 95\% CI 1.66-5.18, $\mathrm{p}=0.0002$ ). In pregnancies where the fetus was $C 1 / C 2$ we could determine parent of origin of the $C 2$ group. In both categorical and continuous analyses, the presence of maternal KIR2DSI only has an effect on birth weight 
Table 1. KIR known to bind HLA-C and their HLA-C ligands.

\begin{tabular}{lccc}
\hline KIR & HLA-C Ligand & KIR Haplotype location & Activating/Inhibitory \\
\hline 2DL1 & C2 & $A$ and some $B$ & Inhibitory \\
2DL2 & C1, some C2 & $B$ & Inhibitory \\
2DL3 & C1 & $A$ & Inhibitory \\
2DS1 & C2 & $B$ & Activating \\
2DS2 & Possibly C1 & $B$ & Activating \\
2DS4 & Some C1 and some C2 & $A$ (often deleted) & Activating \\
\hline
\end{tabular}

Table 2. Presence of maternal KIR2DSI associates with increased birth weight in both categorical and continuous analysis $\mathrm{n}=1316$. This is enhanced when the fetus has more HLA-C2 epitopes than the mother $\mathrm{n}=304$ and specifically paternally derived $C 2 \mathrm{n}=204$. Data summarised from tables I, II, and IV-VII in Hiby $\mathrm{S}$, Apps R, Chazara O, et al. Maternal KIR in combination with paternal HLA-C2 regulate human birth weight. J. Immunol 2014; 192: 5069-5073.

\begin{tabular}{|l|cc|ccc|}
\hline & \multicolumn{2}{|c|}{ Continuous analysis } & \multicolumn{3}{|c|}{ Categorical analysis } \\
& Weight & p-value & OR & $95 \% \mathrm{CI}$ & $\mathrm{p}$-value \\
\hline Presence of maternal KIR2DS1 & $78 \mathrm{~g} \uparrow$ & 0.005 & 1.38 & $1.07-1.79$ & 0.01 \\
\hline Maternal KIR2DS1 with more $C 2$ in fetus than mother & $245 \mathrm{~g} \uparrow$ & 0.0002 & 2.93 & $1.66-5.18$ & 0.0002 \\
\hline Maternal KIR2DS1 with paternally derived fetal $C 2$ & $196 \mathrm{~g} \uparrow$ & 0.016 & 2.65 & $1.33-5.26$ & 0.005 \\
\hline
\end{tabular}

when the fetal $C 2$ is paternally derived (paternal $C 2$ $\mathrm{p}=0.016$, maternal $C 2 \mathrm{p}=0.75$ ) (Table 2).

Our findings using MoBa subjects have replicated the association of KIR and HLA-C with poor placentation (pre-eclampsia, and low birth weight) and confirm the importance of maternal KIR/fetal HLA-C interactions in determining the outcome of pregnancy. Of importance is that we now also show an effect in high birth weight pregnancies, implicating a role in the regulation of placentation in normal or excessive invasion. This effect has a clear direct impact on birth weight as a continuous variable, with an effect comparable or even greater than smoking during pregnancy, high altitude and sex of the baby [21-24].

KIR2DS1 is expressed by uterine NK cells and is functional although ascertaining exactly how NK cells operate to subtly define the extent of arterial transformation by trophoblast is an exciting challenge for the future. Nonetheless our findings indicate that a balance of KIR inhibitory and activating stimuli is necessary for optimal trophoblast invasion. NK derived cytokines such as GM-CSF, released in response to activation of KIR2DS1 by binding C2, are one possible mechanism [13].

Not all women with $K I R A A$ genotypes and fetal $C 2$ have a pregnancy disorder. We are now selecting patients from the MoBa cohort to study women who have recurrent pre-eclampsia as well as those who have a normal pregnancy followed by a pre-eclamptic pregnancy and vice versa. We aim to focus on KIR2DL1, the inhibitory KIR for HLA-C2 because there are 4 different KIR2DL1 alleles in the Norwegian population. Our prediction is that particular KIR2DLI alleles will confer most risk.

Perhaps in the long term KIR/HLA-C genotyping might be a genetic predictor of birth weight to identify those at risk of FGR, pre-eclampsia or macrosomia.

\section{ACKNOWLEDGEMENTS}

Figure 1 reproduced with kind permission of the Journal of Immunology. This work was supported by Wellcome Trust Grants 090108/Z/09/Z and 085992/Z/08/Z, British Heart Foundation Grant PG/09/077/27964, and the Centre for Trophoblast Research. This work was also supported by Frederick National Laboratory for Cancer Research Contract HHSN261200800001E and by the Intramural Research Program of National Institutes of Health, Frederick National Laboratory, Center for Cancer Research. The content of this publication does not necessarily reflect the views or policies of the Department of Health and Human Services, nor does mention of trade names, commercial products, or organizations imply endorsement by the U.S. Government. The funders had no role in study design, data collection and analysis, decision to publish, or preparation of the manuscript.

\section{REFERENCES}

1. Moffett A, Loke C. Immunology of placentation in eutherian mammals. Nat Rev Immunol 2006; 6: 584-594.

2. Burton GJ, Woods AW, Jauniaux E, Kingdom JC. Rheological and physiological consequences of conversion of the maternal spiral arteries for utero-placental blood flow during human pregnancy. Placenta 2009; 30 (6): $473-82$.

3. Brossens I, Pijnenborg R, Vercruysse L, Romero R. The "Great Obstetrical Syndromes" are associated with disorders of deep placentation. Am J Obstet Gynecol 2011; 204 (3): 193-201. 
4. Pjinenborg R, Vercruysse L, Hassens M. The uterine spiral arteries in human pregnancy: facts and controversies. Placenta 2006; 27 (9-10): 939-58.

5. Fuchs F, Bouyer J, Rozenberg P, Senat MV. Adverse maternal outcomes associated with fetal macrosomia: what are the risk factors beyond birthweight? BMC Pregnancy Childbirth 2013; 13: 90.

6. Siggelkow W, Boehm D, Skala C, Grosslercher M, Schmidt M, Koelbl H. The influence of macrosomia on the duration of labor, the mode of delivery and intrapartum complications. Arch Gynecol Obstet 2008; 278 (6): 547-53.

7. Oral E, Cağdaş A, Gezer A, et al. Perinatal and maternal outcomes of fetal macrosomia. Eur J Obstet Gynecol Reprod Biol 2001; 99: 167-71.

8. Cheng Y, Norowitz E, Caughey A. The relationship of fetal position and ethnicity with shoulder dystocia and birth injury. Am J Obstet Gynecol 2006; 195: 856-62.

9. Stotland N, Caughey A, Breed E, Escobar G. Risk factors and obstetric complications associated with macrosomia. Int J Gynecol Obstet 2004; 87: 220-6.

10. Hiby S, Apps R, Chazara O, et al. Maternal KIR in combination with paternal HLA-C2 regulate human birth weight. J Immunol 2014; 192: 5069-73.

11. Hiby S, Apps R, Sharkey A, et al. Maternal activating KIRs protect against human reproductive failure mediated by fetal HLA-C2. J Clin Invest 2010; 120: 4102.

12. Moffett-King A. Natural killer cells and pregnancy. Nat Rev Immunol 2002; 2: 656-63.

13. Xiong S, Sharkey AM, Kennedy PR, et al. Maternal uterine NK cell activating receptor KIR2DS1 enhances placentation. J Clin Invest 2013; 123: 4264-72.

14. Moffett A, Colucci F. Uterine NK cells: active regulators at the maternal-fetal interface. J Clin Invest 2014; 124 (5): 1872-79.

15. Colucci F, Boulenouar S, Kieckbusch J, Moffett A. How does variability of immune system genes affect placentation? Placenta 2011; 32: 539-45.

16. Gonzalez-Galarza FF, Christmas S, Middleton D, Jones AR. Allele frequency net: a database and online repository for immune gene frequencies in worldwide populations. Nucleic Acid Res 2011; 39: D913-9.

17. Colonna M, Borsellino G, Falco M, et al. HLA-C is the inhibitory ligand that determines dominant resistance to lysis by NK1- and NK2-specific natural killer cells. PNAS 1993; 90: 12000-4.

18. Hiby SE, Walker JJ, O'shaughnessy KM, et al. Combinations of maternal KIR and fetal HLA-C genes influence the risk of preeclampsia and reproductive success. $J$ Exp Med 2004; 200: 957-65.

19. Hiby SE, Regan L, Lo W, et al. Association of maternal killer-cell immunoglobulin-like receptors and parental HLA-C genotypes with recurrent miscarriage. Human Reprod 2008; 23: 972-6.

20. Magnus P, Irgens LM, Haug K, Nystad W, Skjaerven R, Stoltenberg C and the MoBa Study Group. Cohort profile: The Norwegian Mother and Child Cohort Study (MoBa). Int J Epidemiol 2006; 35: 1146-50.

21. Haddow JE, Knight GJ, Palomaki GE, Kloza EM, Wald NJ. Cigarette consumption and serum cotinine in relation to birthweight. Br J Obstet Gynaecol 1987; 94 (7): 678-81.

22. Jensen GM, Moore LG. The effect of high altitude and other risk factors on birthweight: independent or interactive effects? Am J Public Health 1997; 87: 1003-7.

23. US Department of Health and Human Services. Women and Smoking: A report of the Surgeon General. 2001: Ch3: 296-297, Ch5: 565.

24. Oken EK, Kleinman KP, Rich-Edwards J, Gillman MW. A nearly continuous measure of birth weight for gestational age using a United States national reference. BMC Pediatr 2003; 3: 6. 